\title{
EXPERIENCIAS Y MEDIDAS SOCIOEDUCATIVAS EN LOS DISPOSITIVOS PENALES JUVENILES
}

\section{EXPERIENCES AND SOCIO-EDUCATIONAL MEASURES IN YOUTH CRIMINAL DEVICES}

\author{
Teresa luri $^{2}$ \\ Centro Universitario Regional Zona Atlántica \\ Universidad Nacional del Comahue \\ Argentina \\ Teresa IURI fliagurmandi@rnonline.com.ar
}

\footnotetext{
$1 \quad$ Una primera versión de este trabajo se expuso en la Conferencia realizada por la autora, en el 3er Encuentro Nacional de la Niñez organizada coordinada por ATE y CTA realizada en Viedma (R.N) en el CURZA de la UNCo. 9 y 10 de Noviembre de 2018. Panel: Avance Punitivo.

$2 \quad$ fliagurmandi@rnonline.com.ar Orcid: https://orcid.org/0000-0002-3308-0911?lang=es

Dependencia Institucional: Centro Universitario Regional Zona Atlántica de la Universidad Nacional del Comahue y del Instituto Sup.Form. Docente $N^{\circ} 25$ Prov. De Buenos Aires .Argentina. IURI, Teresa.2017. "Aproximación a la compleja vida de un grupo de "Ni Ni" en Editorial Boletín Redipe Virtual. Revista de la Red Iberoamericana de Pedagogía. Indexada en Dialnet. Volumen $6 N^{\circ}$ 7. Pedagogía, subjetividad y afectividad. Julio de 2017. ISNN 22561536 Págs. 47/ 61. Cali. Colombia

. http://www.rediberoamericanadepedagogía.com/ content/13-revista

IURI, Teresa- Ibañez, Guadalupe M..2017. Estudiar y Trabajar.Políticas, Tensiones, Sentidos y Estrategias". Editorial Académica Española, ISBN 978-620-2-23967-7.Idioma Español. 89 págs.

IURI, Teresa -SÚS, M.C. 2018. elaboración del Módulo "MEDIACIONES PEDAGÓGICAS QUE FACILITAN EL APRENDIZAJE". Serie Monográfica ISSN: 2618-477X 70 págs.

Teresa IURI fliagurmandi@rnonline.com.ar
}

\section{RESUMEN}

En 2015 se realizó un relevamiento nacional sobre adolescentes en conflicto con la Ley Penal. La metodología utilizada fue un censo, que se realizó de manera simultánea en todas las jurisdicciones del país, mediante instrumentos de recolección de datos que fueron suministrados por informantes clave de cada jurisdicción. El Informe con los resultados obtenidos es una publicación realizada en forma conjunta por la Secretaría Nacional de Niñez, Adolescencia y Familia (SENAF) y el Fondo de la Naciones Unidas para la Infancia (UNICEF) y se tituló "Relevamiento Nacional sobre Adolescentes en conflicto con la Ley Penal Año 2015". En el segundo capítulo se examinaron experiencias y medidas socioeducativas puntuales en la gestión de los Dispositivos Penales Juveniles 
bajo la órbita de la SENAF, con sede en la Ciudad Autónoma de Buenos Aires y de seis provincias representativas de las distintas regiones de la Argentina. Desde el ámbito educativo interesa conocer cómo se significan las experiencias y medidas educativas para este grupo de niños y jóvenes atento el marco de leyes que los protegen. Se analizan desde una mirada socio pedagógica. La Pedagogía construye una mirada pedagógica especializada. A través de este trabajo se espera dar cuenta del recorrido legislativo y de intervenciones que seis provincias han ido transitando durante los últimos diez años, y con la convicción que la socialización de buenas prácticas socioeducativas obrará como un estímulo al mejoramiento educativo del Sistema Penal Juvenil en otras provincias. Las mismas deben orientarse a trazar con cada joven un escenario que lo aleje de la transgresión de la norma penal.

PALABRAS CLAVE: experiencias- medidas socioeducativas-dispositivos penales-

\section{SUMMARY}

In 2015, a national survey was conducted on adolescents in conflict with the Criminal Law. The methodology used was a census, which was carried out simultaneously in all jurisdictions of the country, using data collection instruments that were provided by key informants from each jurisdiction.

The report with the results obtained, is a publication jointly produced by the National Secretariat for Children, Adolescents and Family (SENAF) and the United Nations Children's Fund (UNICEF) and was titled «National Survey on Adolescents in conflict with the Criminal Law Year 2015 «. In the second chapter, experiences and specific socio-educational measures were examined in the management of Juvenile Penal Devices under the SENAF orbit, with headquarters in the Autonomous City of Buenos Aires and six representative provinces of the different regions of Argentina. From the educational field, it is interesting to know how the experiences and educational measures are meant for this group of children and young people attentive to the framework of laws that protect them. They are analyzed from a sociopedagogical perspective. Pedagogy builds a specialized pedagogical look. Through this work it is expected to give an account of the legislative journey and of interventions that six provinces have been going through during the last ten years, and with the conviction that the socialization of good socio-educational practices will act as a stimulus to the educational improvement of the Juvenile Penal System in other provinces. They should be oriented to trace with each young person a scenario that moves him away from the transgression of the criminal norm.

PALABRAS CLAVE: experiences - socioeducational measures-criminal devices-

\section{CONSIDERACIONES BÁSICAS}

En 2015, se realizó un relevamiento nacional sobre adolescentes en conflicto con la Ley Penal. La metodología utilizada fue un censo $^{3}$ que se realizó de manera simultánea en todas las jurisdicciones del país, representando la foto de un día (4 de marzo de 2015) de la población de adolescentes alojada en la totalidad de dispositivos penales juveniles, o incluidos bajo programas de supervisión en territorio, mediante instrumentos de recolección de datos que fueron suministrados por informantes clave de cada jurisdicción.

El Informe con los resultados obtenidos, es una publicación realizada en forma conjunta por la Secretaría Nacional de Niñez, Adolescencia y Familia (SENAF) y el Fondo de la Naciones Unidas para la Infancia (UNICEF) en el marco 3 Participaron del trabajo en diferentes funciones: Secretario Nacional de Niñez, Adolescencia y Familia. Subsecretaría de Derechos para la Niñez, Adolescencia y Familia. Director Nacional para Adolescentes Infractores a la Ley Penal. Responsables Técnicos del Proyecto Sabrina Gerosa (SENAF) y Mariángeles Misuraca (UNICEF) y un equipo de investigación, procesamiento y redacción. 
del Proyecto "Actualización de Datos 2015, Descripción de Experiencias y Estándares Acordados en Materia Penal Juvenil" y se tituló "Relevamiento Nacional sobre Adolescentes en conflicto con la Ley Penal Año 2015". Consta de un Prólogo, la Presentación, IV Capítulos y un Anexo en 188 páginas, editado por el Ministerio de Desarrollo Social de la Nación y el Fondo de las Naciones Unidas para la Infancia (UNICEF), impreso en Argentina Primera Edición, Diciembre 2015 .

Desde el ámbito educativo interesa conocer cómo se transitan y se significan las experiencias y medidas socio educativas para este grupo de adolescentes, atento el marco de Leyes que las protegen y como se resguarda el ejercicio de otros derechos. Se analizan desde una mirada socio pedagógica.

La Pedagogía construye una mirada pedagógica especializada. Es una representación mental que se hace el Pedagogo de su actuación en cuanto profesional de la educación. Es la expresión de la visión crítica que tiene el pedagogo de sus métodos y de sus actos. Mentalidad pedagógica específica y mirada pedagógica especializada confluyen en la intervención. Así la relación educativa se vincula a la educación de la libertad y en consecuencia de los derechos y deberes de los sujetos; es una formación en valores y siempre es una actuación comprometida no neutral, que se propone capacitar al educando para decidir y realizar su proyecto personal de vida, en un particular contexto social. (Touriñon López, 2014).

En el segundo capítulo del Informe antes mencionado, se analizaron experiencias puntuales de gestión de los Dispositivos Penales Juveniles bajo la órbita de la Secretaría Nacional de Niñez, Adolescencia y Familia (SENAF), con sede en la Ciudad Autónoma de Buenos Aires y de seis provincias representativas de las distintas regiones de la Argentina: Entre Ríos, Salta, Tucumán, Mendoza, Chubut y Provincia de Buenos Aires. Las provincias relatan sus propias experiencias: su recorrido legislativo, los dispositivos implementados, las intervenciones técnicas, las medidas socioeducativas y los procesos de transformación transitados en la última década, teniendo en cuenta sus diferentes realidades.

La selección de las provincias se realizó a partir de la consideración de tres variables: provincias que pertenecieran a las distintas regiones del país; que tengan en funcionamiento distintas modalidades de intervención en dispositivos de aplicación de una medida judicial; y que hubieran avanzado, aunque de manera parcial y con dificultades, en el principio de especialización.

La Secretaría Nacional de Niñez, Adolescencia y Familia es el órgano regidor de las políticas públicas de niñez, adolescencia y familia en el ámbito nacional. Ha realizado diversas investigaciones y análisis de la situación de los dispositivos penales juveniles, a los efectos de reunir información que permita impulsar una mayor adecuación de los mismos a los estándares nacionales e internacionales en materia de política penal juvenil.

Aesos efectos, define el concepto de dispositivo penal juvenil como "una modalidad particular de organización de las intervenciones del Estado para implementar una medida judicial como respuesta a la infracción, o presunta infracción, de la ley penal por parte de una persona menor de edad" (Rel. 2015, pág.11) ${ }^{4}$

Los dispositivos penales juveniles registrados fueron: el Programa de Supervisión y Monitoreo de Jóvenes en el Ámbito Socio comunitario, las Residencias Socio-educativas, y los Centros Socioeducativos de Régimen Cerrado.

$4 \quad$ Relevamiento Nacional sobre Adolescentes en conflicto con la Ley Penal Año 2015. "Actualización de Datos 2015, Descripción de Experiencias y Estándares Acordados en Materia Penal Juvenil". SENNAF-UNICEF. En adelante se citará (Rel.2015. pág...) 
La Secretaría Nacional de Niñez y Adolescencia tiene a cargo la formulación, coordinación y ejecución de políticas, programas y medidas destinadas a prevenir el delito juvenil, asegurando los derechos y garantías de los adolescentes infractores a la Ley Penal. En tanto las Secretarías de Niñez y Adolescencia de cada Jurisdicción, tienen a su cargo las funciones orientadas a la promoción y protección integral de los derechos del niño.

En este trabajo interesa destacar las relacionadas con la protección de los derechos de los

adolescentes y jóvenes, en conflicto con la ley penal, en especial el derecho a la educación, en instituciones de encierro. ${ }^{5}$ En la actualidad, solo ingresan a los dispositivos penales cerrados jóvenes de 16 y 17 años con medidas privativas de libertad, dispuestas por la justicia penal

Del análisis del perfil sociodemográfico de estos jóvenes surge que, a pesar de que el delito y la transgresión atraviesan todos los sectores sociales, las instituciones de encierro están destinadas a pobres y excluidos, sujetos con múltiples privaciones y conculcación de derechos. Su bajo nivel educativo evidencia, entre otras cuestiones, que su paso por el sistema educativo fue breve y frustrante. La modalidad Educación en Contexto de Encierro, y el plan FiNes (de finalización de estudios primarios y secundarios), entre otras Políticas Educativas de los últimos años apuntan a garantizar el ejercicio del derecho a la educación, fundante de otros derechos. La educación es el derecho humano a través del cual las personas conocen todos los derechos humanos. Además desarrolla la personalidad en forma integral.

Así, los Principios Básicos y las Reglas Mínimas para el tratamiento de las personas privadas de

$5 \quad$ Derecho prescripto por la Constitución Nacional y Provinciales; Ley Nacional de educación 26.206 y Leyes de Educación Jurisdiccionales y por la Ley $N^{\circ} 26.695$ de Ejecución de la Pena Privativa de la Libertad, modificatoria de la $N^{\circ}$ 24.660; en particular los artículos vinculados a la educación de los estudiantes privados de su libertad. libertad establecen el derecho de los internos a participar en actividades culturales y educativas encaminadas a desarrollar plenamente la personalidad humana. De manera que todas las medidas en los dispositivos deben ser socioeducativas. Tanto en el contexto de educación formal, como en el contexto más amplio de la convivencia, en el dispositivo. Según Paulo Freire,

“... las condiciones materiales del espacio pueden ser o no ser en sí mismas pedagógicas"........ y como no hay espacio sin tiempo, entonces el tiempo pedagógico es el otro elemento constitutivo de la situación educativa"... "Obviamente el tiempo educativo está al servicio de la producción de saber... la pregunta que debemos hacernos es: al servicio de quiénes, de qué ideales producimos, conjuntamente con los alumnos, el saber..." (Freire, 2003, pp.34/35).

Nos preguntamos ¿Se utiliza este tiempo $y$ espacio a favor de los intereses de los adolescentes? Porque la educación construye al sujeto capaz de conocer y pensar en sus derechos, asumir sus deberes, y contraer compromisos.

Destacamos además, que las SENAF generan los ámbitos para la ejecución de medidas

socio educativas que centradas en la responsabilidad del joven infractor, posibiliten su real inserción en su comunidad de origen; atienden y controlan el estado y las condiciones de alojamiento; formulan los programas y dispone las mejores prácticas de protección de derechos, en función de los avances teóricos y prácticos en materia de Responsabilidad Penal Juvenil; promueven la participación de la familia en el proceso de resocialización de cada joven. Otra tarea es coordinar con la autoridad judicial competente, las medidas de protección especial de derechos y las medidas restrictivas 
y privativas de libertad, impuestas en el marco de los procesos penales. Son responsables de la elaboración y presentación de informes periódicos para dar cuenta de la evolución y los resultados de las medidas socioeducativas, impuestas a cada joven procesado.

A nivel federal en la materia, el Consejo Nacional de Infancias, Adolescencias y Familias, integrado por los Secretarios de Infancias, Adolescencias y Familias y por el Secretario Nacional, se reúne periódicamente y han suscripto Actas de Compromiso: Actas: "Una política respetuosa de los Derechos Humanos para los Adolescentes infractores de la Ley Penal", 18/04/2008; Acta "Hacia una mayor Calidad Institucional de los Dispositivos Penales Juveniles.",20/05/2011; Acta "Por una Ley Penal Juvenil acorde a los parámetros y directrices complementarios de la Convención Internacional sobre los Derechos del Niño". 24/10/2014; Acta Declaración "Contenidos mínimos de una nueva Ley Penal Juvenil".17/07/2015

Como técnica de indagación de las experiencias, la SENNAF utilizó las entrevistas, $y$ observaciones en terreno. Entrevistas con funcionarios provinciales según un protocolo pero que produjo un intercambio de perspectivas; y realizó visitas a los dispositivos, y se emplearon entrevistas con los equipos técnicos de los mismos.

A través de este trabajo, y tomando como base el cap. Il del informe del Relevamiento Nacional sobre Adolescentes en conflicto con la Ley Penal Año 2015 se espera dar cuenta del recorrido legislativo y de intervención técnica que cada territorio ha ido transitando durante los últimos diez años, atendiendo las singularidades de cada región, y con la convicción que la socialización de buenas prácticas socioeducativas obrará como un estímulo al mejoramiento del Sistema Penal Juvenil en otras provincias. Se consignan, avances y desafíos pendientes.
Se incluye en el PROIN Programa de Investigación Las prácticas educativas. Abordaje desde las experiencias y significaciones de jóvenes y adultos Código 04/V099, PI Jóvenes $y$ adultos. Diversos modos de transitar $y$ significar las experiencias educativas en el nivel medio aprobado por Ordenanza № 0905/17 del Consejo Superior UNCo. María Inés Barilá, Directora. Asesora Teresa luri.

\section{ANÁLISIS E INTERPRETACIÓN DE EXPERIENCIAS}

El análisis e interpretación de las experiencias de gestión de dispositivos penales juveniles sistematizadas en el ámbito de la Secretaría Nacional de Niñez, Adolescencia y Familia, con la participación de la ciudad autónoma de Buenos Aires, y seis provincias, permite identificar buenas prácticas, de promoción y protección de los derechos de adolescentes en conflicto con la Ley Penal.

Algunas acciones son compartidas por las provincias, con características particulares de acuerdo a su realidad, en cambio otras son propias de cada una de ellas y podrían ser replicadas en otras, atento los buenos resultados obtenidos.

En todas las provincias que participaron, el proceso de transformación de los últimos diez años ha buscado ampliar, la accesibilidad de derechos en materia penal juvenil. El marco normativo general de esta transformación lo constituyen los tratados internacionales en materias de Derechos Humanos contenidos en la Constitución Nacional, la Constitución Nacional y, en particular, la Ley $\mathrm{N}^{\circ} 26.061 \mathrm{de}$ Protección Integral de Derechos de los Niños, Niñas y Adolescentes y la aún vigente Ley $\mathrm{N}^{\circ}$ 22.278 (Régimen Penal de Menores) limitada por el conjunto normativo de referencia. En particular, la Convención de los Derechos del Niño (Artículos 37 y 40), leyes y resoluciones específicas, y jurisprudencia pertinente. 
Desdelaadopción de la Convención Internacional de los Derechos del Niño y en particular desde la sanción en el año 2005 de la Ley Nacional de Protección Integral de los Derechos de Niños, Niñas y Adolescentes 26.061, en particular, las provincias han sancionado sus propias Leyes de Protección de los Derechos de Niños, Niñas y Adolescentes.

Han avanzado en la implementación de un Sistema Penal Juvenil conforme a los Derechos Humanos, sentando criterios específicos y claros para la intervención del Estado en este campo. Crearon organismos y dispositivos, articulando su funcionamiento con otras instituciones y organismos tanto públicos como privados y mejorando las prácticas al interior de esos dispositivos.

Desde la mirada pedagógica se seleccionaron aquellas medidas socioeducativas que estimamos mejor contribuirían a lograr la finalidad de acompañar al joven en el proceso de la elaboración de un proyecto de vida que lo sostenga alejado de situaciones conflictivas que impliquen peligro.

\section{RECORRIDOS TRANSITADOS}

Se identificaron algunas medidas comunes a todas las provincias seleccionadas:

- Han encabezado un fuerte proceso de adecuación de las Políticas Públicas con el propósito de revertir el modelo de la Situación Irregular, implementando uno basado en el paradigma de la Protección Integral de Derechos. Pese a que, a través de la vigencia de la Ley $\mathrm{N}^{\circ} 22.278 / 80$ de Régimen Penal de Minoridad, "persiste un fuerte sesgo tutelar con características de protección", que determina todavía la discrecionalidad de los jueces (Rel.2015, p. 65)
- Han creado sitios- que reciben diferentes denominaciones- los Centros de Admisión, Centro de Diagnóstico/ Centro de Diagnóstico y Derivación/Centro de atención de jóvenes/Áreas de admisión/ Centros de Recepción, para evitar que los adolescentes sean alojados en las comisarías. Para el caso en que un adolescente fuera aprehendido por la autoridad policial $-\mathrm{u}$ otra fuerza de seguridad dependiente del Poder Ejecutivo Nacional- es importante que sea inmediatamente trasladado a los mismos para evitar el ingreso y la permanencia de los adolescentes en comisarías, en concordancia con el Principio de Especialidad ${ }^{6}$

- Se ha avanzado significativamente al diferenciar las medidas de protección de las sancionatorias. Sobre todo, en impedir la privación de libertad como una medida de "protección" de derechos, o según su situación de vulnerabilidad social.

- En ese sentido, también hay límites al ingreso de adolescentes no punibles al Sistema Penal Juvenil?.

- En todas las provincias seleccionadas, la privación de libertad es considerada como última ratio (último recurso).

- El lineamiento de los principios básicos de especialización, excepcionalidad, accesibilidad de Derechos y la promoción de medidas socioeducativas tendientes al cumplimiento de la sanción en territorio, en todas las provincias participantes, representan un avance significativo.

$6 \quad$ Convención Americana de Derechos Humanos (CADH), art. 5.5; CDN, art. 40, inc. .3; Reglas de Beijing, regla 2.3; Directrices de Acción sobre el niño en el sistema de justicia penal, directrices 13.d y 14.a y d.; Reglas de Beijing, (art. 4.1) regla 16; Reglas de Tokio, regla 7; Reglas de Beijing, regla 22; Directrices de Acción sobre el niño en el sistema de justicia penal, directriz 24; y Directrices de Riad, directriz 58 ; Comité de los Derechos del Niño, Observación General No 10 (2007), Los derechos del niño en la Justicia de Menores, pfo. 33

$7 \quad$ Resolución SENAF 313/2015. 
- Se intenta con diferentes resultados resguardar la especialización hasta la etapa de ejecución penal incluida.

Los avances en el acceso a los derechos en el interior de los dispositivos penales juveniles han buscado asegurar un principio central: -ya decíamos en otro momento luri, 20178- que la privación de libertad no vulnere otros derechos de los adolescentes (a la educación, a la salud, a las actividades recreativas, religiosas $y$ culturales, al deporte).

- En esta línea, se registra que en las seis provincias y en la ciudad autónoma, se incrementaron las actividades educativas y de formación en los centros de régimen cerrado y aumentaron los niveles de escolaridad de los adolescentes incluidos. Esto refleja el avance de la educación formal a través de la "Modalidad Educación en Contextos de Encierro" (Ley Nacional 26.206/06) y el plan FinES (Plan Nacional de Finalización de Estudios Primarios y Secundarios); junto con otras intervenciones no formales al interior de estas instituciones. En general abocados a la escolarización primaria y secundaria, que consiste en la concurrencia de los jóvenes al espacio escolar de cinco horas diarias durante la mañana. Por la tarde, se desarrollan los talleres de educación no formal.

- Brindan también escenarios de aproximación laboral y espacios de formación para el trabajo, en mayor o menor medida todas las provincias seleccionadas.

Estas actividades de formación y capacitación laboral amplían las posibilidades de inclusión

$8 \quad$ "Si el niño está en conflicto con la ley parece ser menos niño y más en conflicto con la ley; al que entra en conflicto con la ley, parece que esto lo excluye de las políticas públicas de protección. Y esto no debe ser así. Los niños poseen los derechos que corresponden a todos los seres humanos $y$, además, tienen un plus de derechos especiales derivados de su condición, a los que corresponden deberes específicos de la familia, la sociedad y el Estado, por ello se han separado los sistemas penales: el Juvenil y el General. en su comunidad al momento del egreso.

Otras medidas socioeducativas son particulares de algunas provincias:

- El criterio del avance de los jóvenes se basa en la progresividad institucional. Se identifica una secuencia de fases que permite agrupar a los jóvenes según la etapa en que cada uno se encuentre dentro del recorrido institucional. Algunas provincias (CABA, Rel. 2015, p.42) las han organizado en etapas diferenciadas. Tales etapas son: ingreso y diagnóstico, convivencia, pre-autonomía y autonomía.

Estas etapas permitirían disponer de tiempos para cada una de ellas; centrarse en la de convivencia para estimular a los jóvenes a avanzar hacia la preautonomía y autonomía.

El camino más adecuado, a nuestro modo de ver, consiste en crear condiciones, desde el ingreso, para que el joven sintiéndose comprendido y aceptado, tome conciencia de la naturaleza y de las consecuencias de sus propios actos. No demorar la etapa del diagnóstico, a los fines de iniciar tempranamente la etapa de convivencia educativa en la institución de encierro. Recordemos que hay distintos tipos de convivencia (familiar, escolar, vecinal, barrial) y no todas "se especifican y cualifican como educativas". La convivencia tiene que especificarse "atendiendo a las condiciones propias de ese espacio", en este caso particular, debe ser "orientada a construir y usar experiencia axiológica para actuar pacíficamente en relación consigo mismo, con el otro y con lo otro"... (Touriñón López, 2014, p.9- p.11). 
- El Proyecto Institucional ${ }^{9}$ en cada Dispositivo (Tucumán. Rel. 2015, p. 65) es otro de los instrumentos básicos para acompañar estos cambios.

Su elaboración, permite desarrollar los objetivos a corto, mediano y largo plazo; establecer los fundamentos para la construcción de una estrategia consensuada para todo joven, trabajando en la articulación interinstitucional, y capitalizando el conocimiento y la experiencia de cada integrante del equipo técnico, que deben posicionarse. Frente a las representaciones devaluadas acerca de estos adolescentes y las disputas en el campo teórico sobre el sentido de educar en contextos de encierro ${ }^{10}$ obligan a tomar posición, tanto para reflexionar como para realizar aportes (luri, 2019).

- En cuanto al dispositivo de Supervisión y Monitoreo de Adolescentes en el ámbito socio comunitario, han intensificado una relación de complementariedad con los operadores del Poder Judicial en las acciones realizadas con los adolescentes, para dar cumplimiento a las medidas dispuestas (CABA, Rel.2015, p.44)

Un mayor acercamiento y la frecuencia del diálogo con las autoridades judiciales han permitido ir ajustando las sugerencias $y$ recomendaciones incluidas en los informes

$9 \quad$ La Pedagogía Institucional tiene una amplia experiencia en la gestión sobre la base de proyectos institucionales. La elaboración del Proyecto Institucional da lugar a generar los espacios para cuestionar y discutir en forma interdisciplinaria, sobre la situación institucional, la vida cotidiana de los adolescentes, y acordar las pautas de intervención, a fin de revertir un paradigma obsoleto y contrario a nuestro ordenamiento jurídico. Acordar modos de trabajo entre los distintos profesionales que intervienen. Fijar objetivos a corto y mediano plazo. Permitiría la generación de aprendizajes y experiencias que permitan fortalecer el pleno ejercicio de su ciudadanía y llevar adelante un proyecto de vida en el seno de la comunidad. Se completaría con el Plan individual de ejecución de la medida.

$10 \quad$ Nos referimos a si se debe educar en estos contextos, ¿para la recuperación? ¿para la rehabilitación? ¿para la resocialización? ¿para la restitución de derechos? IURI, 2019. técnicos de los equipos ${ }^{11}$. También sería muy importante hacer una devolución a los adolescentes sobre su desempeño dentro de la institución y su avance en las distintas fases.

- Por este motivo, se ha avanzado en la articulación con el Poder Judicial, ( Tucumán, Rel. 2015, p.70) para generar un Protocolo de Ingreso de Adolescentes a Dispositivos Penales, que establece con claridad los tipos de Dispositivos Penales Juveniles existentes, sus objetivos y los casos que pueden ser abordados por cada uno. Dicho Protocolo, fue aprobado en 2015 por la Corte Suprema de Justicia de esa Provincia.

El mismo debe darse a conocer a los adolescentes, y asegurarse que lo comprendan.

- También se ha articulado la inclusión de los jóvenes en el mundo laboral con los Ministerios de Trabajo. Se han promovido protocolos de coordinación interinstitucional con Hospitales Públicos, o Salas de Primeros Auxilios para favorecer la derivación de los adolescentes para el cuidado de la salud y odontológico, y la de los de consumo problemático. (Tucumán, Rel. 2015, p.70).

- Asimismo, la constitución de sedes territoriales ha permitido expandir el ámbito de corresponsabilidad social en la intervención, habilitando espacios de experiencia socioeducativa para los adolescentes, con sus familias y la comunidad. La frecuencia de las visitas asciende a 4 por semana, con una duración de 2 horas cada una. Incluso en aquellos casos en que las familias tuvieran que viajar porque viven lejos del Centro se

\footnotetext{
11 Los instrumentos que se podrían poner a disposición de los Jueces de menores para facilitar esta supervisión: Plan individual de ejecución de la medida, Informes periódicos de seguimiento, Partes de incidentes elaborados sobre la base de la aplicación del Reglamento Disciplinario, solicitud de permisos... y cualquier otro que sea solicitado a lo largo de la ejecución de la medida.
} 
facilitan recursos económicos ${ }^{12}$, desde el órgano administrativo. Además, pueden realizar llamados telefónicos todos los días. (Chubut, Rel. 2015, p.90).

- La Coordinación de Educación en Contexto de Encierro del Ministerio de Educación de la Provincia de Salta, organizó una sala de alfabetización digital, en uno de los dispositivos, y nombró a dos docentes - un profesor de Informática y otro de Sistema de redes - equipada con computadoras de última generación; un punto de acceso digital, con internet inalámbrico (WiFi) gratuito; con micro cine, contenidos audiovisuales y consolas de videojuego (hasta el momento del informe, primera en desarrollarse) (Rel. 2015, p. 57 ).

- En otro dispositivo de Orán, Salta, se recibe mensualmente el Programa de Cine Móvil y la religión Católica Apostólica Romana participa sistemáticamente con diversas actividades, y las confesiones evangelistas efectúan actividades esporádicas. Actividades religiosas se llevan a cabo también en otras de las provincias participantes.

- En Mendoza, las actividades de esparcimiento consisten en ver televisión, escuchar música 0 entretenimientos, como metegol y mesa de ping pon, que se desarrollan en los espacios comunes. La institución posee libros y revistas y bibliotecas itinerantes para leer e informarse. Parte de la rutina de los jóvenes está marcada por el mantenimiento de los sectores (limpieza, cuidado del mobiliario, cuidado del jardín, aseo de prendas personales, entre otros). En los espacios verdes también se han implementado pequeños viveros. Favorecidos con cursos de capacitación del Instituto Nacional de Tecnología
Agropecuaria (INTA) como así también cursos de herrería, de marroquinería y de plástica. (Rel. 2015, p.82)

- En todas las provincias se promueven equipos técnicos interdisciplinarios (médicos, psicólogos, psiquiatras, psicopedagogos y trabajadores socialesentre otros-), y en algunas, la Secretaría de Derechos Humanos, u organismos equivalentes participan en esa materia, en la formación y capacitación de los integrantes de los Equipo Técnicos Interdisciplinarios (Rel. 2015, p.70) ${ }^{13}$.

Resta convocarlos en otras provincias. Llama la atención que tratándose de medidas socioeducativas no se incluyan pedagogos en los equipos.

- Se ha solicitado también, la intervención del Programa de Arquitectura y Servicios - dependiente del Ministerio de Derechos Humanos-para que elabore las propuestas edilicias pertinentes (Salta. Rel. 2015, p. $56)$.

- En la (CABA, Rel.. 2015, p. 42) algunos dispositivos son mixtos y en consecuencias todas las actividades en el establecimiento son mixtas, de modo que los y las jóvenes comparten los espacios del comedor, la escuela, talleres, actividades deportivas y de recreación.

- La provincia de Mendoza para contar con procedimientos disciplinarios compatibles con el respeto y la dignidad de los adolescentes, ha elaborado un Manual de Procedimiento de la Unidad de Internación con un régimen de sanciones para los jóvenes alojados, en el que se determinan

13 Se ha hecho un gran esfuerzo para que el resto de los actores del Sistema Penal -especialmente el Poder Judicial- comprenda que si la capacidad de los Dispositivos de privación de libertad se excede, se pierde el sentido de la intervención (Tucumán. Rel. 2015, p70). 
las pautas de comportamiento indebidas dentro del establecimiento.

Porque se da por sentado que los jóvenes sabrán como comportase en el establecimiento olvidando que precisamente son jóvenes que han tenido conflicto con el ajuste a las normativas.

- Asimismo, se explicita la prohibición de aplicación de encierro y otras disposiciones por parte de los operadores. La aplicación de estas sanciones graves de manera excepcionalísima solo puede ser dispuesta por el Director, en exclusiva protección los jóvenes y con inmediata noticia al Juez Penal de turno, quien debe decidir sobre la viabilidad de la medida (Rel. 2015, p. 84).

- En Chubut se han implementado otras medidas alternativas por fuera de la órbita del Poder Ejecutivo, a través de la Justicia Restaurativa, a través de la metodología de mediación, reparación, pagos, pedidos de disculpas y realización de tareas comunitarias, entre otras ( Rel. 2015, p. 89).

- En Entre Ríos (Rel.2015, p. 51) decidida una intervención, se firma un Acta Compromiso con todos los involucrados, la cual debe ser la herramienta legal a implementar, y cuya finalidad es acordar el plan de acción. Esta debe ser firmada en forma conjunta por el joven, su responsable adulto y por personal del Programa interviniente. En el ámbito interno, el Acta contribuye a sistematizar la intervención, plantear objetivos claros y evitar la superposición de funciones.

Asimismo, permitirá revisar los acuerdos realizados con el objeto de reafirmarlos o redirigirlos.

- En la prov. de Bs.As. el ReINA (Registro Integral de Niños y Adolescentes en el Sistema de la Responsabilidad Penal Juvenil de la Provincia de Buenos Aires) es un nuevo sistema online que busca optimizar el monitoreo y la evaluación de los procesos por los que atraviesan las y los jóvenes durante el cumplimiento de medidas judiciales.

- La Secretaría de Niñez y Adolescencia provee a cada familia viáticos para dos personas y alojamiento, si fuere necesario, para que puedan efectuar una visita semanal. Asimismo, se contemplan visitas eventuales por situaciones particulares (Rel.2015, p.100).

Digitalizar los legajos de las y los jóvenes, al igual que las intervenciones que se realicen, facilita el acceso a la información y a las estadísticas. Esto permite contar con información que beneficiará el trabajo cotidiano y el seguimiento y evolución de cada joven. También para evaluar la ejecución de las Políticas Públicas.

En síntesis, además de las medidas socioeducativas evaluadas, en general en todas las provincias los adolescentes participan en una o algunas de las actividades de programas socioeducativos, laborales, culturales, recreativos, y en proyectos, para propiciar y desarrollar sus intereses. Se trabaja en viveros, con radios, canales de televisión, institutos de arte, y se coordina con las municipalidades para que los jóvenes puedan exponer sus trabajos en lugares, como la casas de la cultura, museos y otros sitios públicos.

Como estas actividades son esporádicas y a veces no se cuenta con los espacios suficientes para todos los internos, en este punto se estima conveniente habilitar un registro de entidades de la comunidad con quienes establecer convenios para realizar estas actividades de una manera más sistemáticas, y facilitar espacios.

Advertimos que se ha iniciado un lento $y$ dificultoso proceso de transformación, con el fin de implementar las Directrices Internacionales y 
Nacionales en materia penal juvenil y redefinir las Políticas Públicas y revisar las prácticas, en el abordaje de los jóvenes en conflicto con la Ley Penal. Sobre la base de los ejes rectores del nuevo paradigma de Protección Integral de los Derechos de Niños, Niñas y Adolescentes, los estados provinciales comienzan a reconsiderar y profundizar las políticas necesarias para llevar adelante esta nueva perspectiva.

Profundizar en la especialización de las medidas socioeducativas en las instituciones que intervienen con los adolescentes infractores o presuntos infractores a la Ley Penal, nos marca un rumbo para cumplir con la finalidad de acompañar al joven en la elaboración de un proyecto de vida que lo sostenga alejado de aquellas prácticas vinculadas con la transgresión a las Leyes Penales.

\section{A MODO DE CIERRE: LOGROS, DESAFÍOS Y OBSTÁCULOS}

Los pensamientos tutelares nos han colonizado en el modo de entender y ocuparnos de los niños por más de 100 años de antigüedad. Modificar esta cultura es hablar de revisar nuestras prácticas, cada uno en su ámbito de competencia, y exige un esfuerzo permanente, pero también una voluntad y un compromiso con el cambio, y para eso hay que estar convencidos de qué hay que cambiar y para qué hacerlo.

Desde esa perspectiva tutelar, el actual Sistema otorga al Juez en lo Penal de Menores la posibilidad de disponer medidas restrictivas y privativas de libertad con un alto grado de discrecionalidad, pero existe un cuerpo de normativas y jurisprudencia nacional $e$ internacional que lo habilitan al cambio.

Las Actas Compromiso asumidas a través del Consejo Federal son un evidente respaldo político e institucional estratégico para lograr los mayores cambios en cuanto al diseño, la implementación y el seguimiento de las Políticas Públicas.

Uno de los propósitos centrales es que los adolescentes reparen el daño causado, realicen actividades comunitarias y se capaciten. Que cada adolescente entienda las consecuencias directas e indirectas de sus actos, asuma la responsabilidad de sus acciones y se vincule con programas que faciliten su desarrollo integral.

Las intervenciones deben hacer hincapié en el proceso de Responsabilización del adolescente infractor o supuesto infractor a la Ley Penal, las consecuencias de su acto, y la construcción de un proyecto de vida acorde con sus necesidades. Lo cual no surge en forma especial en el relato de experiencias.

Medidas alternativas como la mediación, conciliación, reparación, conferencia familiar, estudio de caso de conferencia familiar grupal, integración e inclusión, escucha en audiencia, distintos roles distintas verdades, requeridos por las normas internacionales (justicia restaurativa $)^{14}$ son poco citadas, con excepción de la prov. de Chubut.

Por ello, la mejora permanente de la calidad institucional continúa siendo una meta lo mismo que la revisión continua de las intervenciones para seguir adecuando las prácticas a una política respetuosa de los derechos humanos de los adolescentes y la construcción de un proyecto de vida acorde con sus necesidades.

También es necesario profundizar la articulación con el ámbito laboral, con el sistema de salud, una coordinación más estrecha entre todos los actores es necesaria.

\footnotetext{
$14 \quad$ La justicia restaurativa incluye el reconocimiento de la responsabilidad por parte del ofensor, aproximación a las personas afectadas por el hecho y actos de restauración de los daños causados. Ofrece alternativas inmediatas de reincorporación al colectivo social, ya que promueve que se enfrente la responsabilidad de quien cometió la ofensa y el miedo de quien la padeció, favoreciendo en ambos una estrategia más adaptativa de resolución de problemas. Hace posible un espacio humano, comunicativo, entre ambos.
} 
Para llevar a cabo las mejoras institucionales y adecuarse a los estándares internacionales en materia Penal Juvenil, se requiere de mayores recursos económicos y humanos, tanto en materia de infraestructura, movilidad, telefonía, tecnología, profesionales, como de presupuesto para becas y capacitación de los jóvenes profesionales, aunque solo la provincia de Mendoza lo requiere. En todas las provincias se necesita de especialización del personal público que trabaja con este sector, en derechos de los adolescentes.

No se ha trabajado el perfil de los directores y operadores de los centros.

Se advierte la falta de implementación de un plan de acción personalizado para el egreso.

Si consideramos el recorrido realizado desde la transformación del marco normativo, hasta el momento, de las adecuaciones institucionales y las prácticas socioeducativas ejecutadas, encontramos algunos avances significativos, pero persisten algunos obstáculos que resultó pertinente compartir.

El desafío es seguir avanzando en la construcción de una mirada crítica hacia el interior de la práctica y sus resultados, en todos los Dispositivos, con el propósito de fortalecer la adecuación en la especialización de las instituciones y la capacitación de sus equipos técnicos.

No surge de este análisis que ninguna de las provincias seleccionadas haya logrado cumplir, hasta la fecha con su responsabilidad de poner en funcionamiento todas las estrategias socioeducativas señaladas como positivas e impulsar políticas y programas específicos orientados a la promoción y protección de derechos. Todavía nuestros niños, adolescentes y jóvenes, necesitan más derechos y no más punitivismo.
Es importante trazar con cada joven un escenario que lo aleje de la transgresión de la norma penal, estimulando sus capacidades y generando responsabilidad en sus actos para hacer efectivo el cumplimiento de todos sus derechos y obligaciones.

Por otra parte, consideramos, que el Estado, sigue estando en deuda respecto de nuestros jóvenes, de realizar políticas públicas, focalizadas que apunten a la prevención del delito y la protección de sus derechos.

Como investigadores universitarios, sostenemos que se necesita más trabajo de campo en las instituciones, con las voces de los adolescentes como protagonistas.

\section{REFERENCIAS}

Barilá, M.I.- Iuri, T. (2012). "Infancias y Adolescencias Silenciadas. Sus derechos en la encrucijada: entre las políticas públicas y las prácticas concretas". Alemania. Edit. Acad. Española.

Beloff, M. Los Sistemas de Responsabilidad Penal Juvenil en América Latina. Publicado en García Méndez Emilio y Beloff, Mary (compiladores) Infancia, Ley y Democracia en América Latina. Análisis crítico del panorama legislativo en el marco de la Convención Internacional de los Derechos del Niño (19901998) Temis / Depalma, Bogotá. 1998.

Bruno R. C. 2015. "Tras un Sistema de Responsabilidad Penal Juvenil para la Provincia de Río Negro: compromiso legal e imperativo convencional". Consultado el 10 de noviembre de 2017 en servicios.jusrionegro. gov.ar/inicio/comunicacionjudicial/index.../545responsabilidad .Actualizado el 7/11/2017.

Comité Argentino de Seguimiento y Aplicación de la Convención Internacional sobre los Derechos del Niño ( 1991) consultado el 8 de noviembre de 2017 de la fuente https:// 
casacidn.org.ar/institucional/quienes-somos

. García Méndez, Emilio y Beloff, Mary (compiladores), Infancia, Ley y Democracia en América Latina. Análisis crítico del panorama legislativo en el marco de la Convención Internacional sobre los Derechos del Niño (1990-199, Temis/ Depalma, Bogotá, 1998.

.Gutiérrez, Mariano H. "La inclusión de la educación dentro de la Ley de Ejecución: un retroceso". Profesor de Criminología (UNL, UP, UB, Azuay).Cepoc- cepoc blogspot/ 2011/08 ley-26. html doctrina 31688 contrextos de encierro. Pdf consultado el $11 / 10 / 2018$

.Disertaciones a cargo del Especialista en Derecho Internacional y DD.HH., Dr. Alejandro Morlachetti y la especialista en Protección (UNICEF, Lic. Manuela Thourte ). XV Jornadas Nacionales FOFECMA - Panel: "Niños, niñas $y$ adolescentes Consultado en fofecma. org/2015/09/ninas-ninos-y-adolescentes-enconflicto-con-la-ley-penal/22 sep. 2015 -

.Freire, P. (2003) El grito manso. Seminario parte 1 y 2. Siglo XXI Ed. Buenos Aires. Argentina. Disponible en pdfhumanidades.com/ sites/default/files apuntes/El Grito Manso.pdf. Consultado el 27 de mayo de 2019.

.luri, T. 2017. "Promoción de nuevas prácticas que reafirman la protección de derechos de adolescentes y jóvenes". Ponencia presentada en las Jornadas "Debates por Nuestra Niñez: Intervenciones en infancia/s y adolescencias/s ¿Políticas postergadas?" Realizadas en el Centro Universitario Regional Zona Atlántica de la UNCo. Durante los días 16 y 17 de noviembre de 2017. .luri, T. 2019. "La intervención pedagógica no puede ser una respuesta tardía". Ponencia presentada en las $\mathrm{VI}$ JORNADAS DE PSICOPEDAGOGÍA DEL COMAHUE. "EI aprendizaje en infancias, adolescencias y juventudes. Prácticas, intervenciones y producción de saberes". Realizadas en el Centro Universitario Regional Zona Atlántica de La UNCo, en Viedma Río Negro, durante los días 15,16 y 17 de mayo de 2019.

“JUSTICIA Y DERECHOS DEL NIÑO”.UNICEF. Fondo de las Naciones Unidas para la Infancia Oficina de Area para Argentina, Chile y Uruguay .MINISTERIO DE JUSTICIA. Comité editorial: Mary Beloff / Miguel Cillero / Julio Cortés / Jaime Cous

. Relevamiento Nacional sobre Adolescentes en conflicto con la Ley Penal Año 2015. Secretaría Nacional de Niñez, Adolescencia y Familia (SENAF). Fondo de las Naciones Unidas para la Infancia (UNICEF) .Responsables Técnicos del Proyecto Sabrina Gerosa (SENAF) y Mariángeles Misuraca (UNICEF).

Situación de niñas, niños y adolescentes sin cuidados ... - Unicef https://www.unicef. org/.../PROTECCION Relevamiento SinCuidadosParentales2015

.Tapia A.-Lobo, A.-Perez, E. 2015. Sistema de Responsabilidad Penal para Adolescentes. Hacia la Protección Integral y la Justicia Restaurativa. Proyecto del Ministerio de Justicia y del Derecho. Universidad de San Buenaventura (Bogotá) Universidad Sergio Arboleda. Universidades Ejecutoras del Proyecto, Bogotá. Colombia. Consultado el 15 de noviembre de 2017, de la fuente https://www.minjusticia.gov. co/.../Sistema\%20de\%20Responsabilidad\%20 Penal\%20pa... 
.Touriñan López, J. M. ( 2014). "La relación educativa es un concepto con significado propio Editorial Revista Virtual Redipe. Año 3 Volumen 6. Págs. 6/41. ISSN 2266-1536.

.UNICEF. 2016. Estado de la situación de la niñez y la adolescencia EN ARGENTINA.

Consultado el 14 de octubre de 2018 de la fuente https://www.unicef.org/argentina/sites/ unicef.org.argentina/files/2018-04/SITAN.pdf

LEYES, REGLAS, DIRECTRICES Y RESOLUCIONES.

Convención Americana de Derechos Humanos (CADH), art. 5.5; CDN, art. 40, inc. .3; Reglas de Beijing, regla 2.3; Directrices de Acción sobre el niño en el sistema de justicia penal, directrices 13.d y 14.a y d.; Reglas de Beijing, (art. 4.1) regla 16; Reglas de Tokio, regla 7; Reglas de Beijing, regla 22; Directrices de Acción sobre el niño en el sistema de justicia penal, directriz 24; y Directrices de Riad, directriz 58 ; Comité de los Derechos del Niño, Observación General № 10 (2007), Los derechos del niño en la Justicia de Menores, pfo. 33

Ley Nº 22.278/1980 Régimen Penal de Menores.

Resolución SENAF 313/2015.

Ley de Protección de Protección Integral de los Derechos de niños, niñas y adolescentes, $\mathrm{N}^{\circ}$ 26.061/ 05.

Ley Nacional de Educación № 26.206/ 06. (ver en especial el Capítulo XII: Educación en Contextos de Privación de Libertad, Art. $55^{\circ}$ a $\left.59^{\circ}\right)$

Ley de Ejecución de la Pena Privativa de la Libertad, N²6.695/2011. De educación en contexto de encierro (sustituye el capítulo VIII, arts. 133 A 142 de la Ley Nac. 24.660/96).

Resolución № 127/10 del CFE donde se aprueba el Documento Base: "La Educación en Contextos de Privación de la Libertad en el Sistema Educativo Nacional"

Anexo de la Resolución № 127/10 CFE donde consta el Documento Base: "La Educación en Contextos de Privación de la Libertad en el Sistema Educativo Nacional" 